the link between GERD and CVD could be attributable to shared risk factors.

Original article Jansson C et al. (2008) Severe symptoms of gastro-oesophageal reflux disease are associated with cardiovascular disease and other gastrointestinal symptoms, but not diabetes: a population-based study. Aliment Pharmacol Ther 27: 58-65

\section{IBD increases the risk of developing arterial thromboembolic disorders}

Previous studies have shown that Crohn's disease and ulcerative colitis increase the risk of venous thrombosis, and that patients with IBD generally are more likely to suffer deep venous thrombosis or pulmonary embolism. The reason for this increased risk is not clear.

In this Canadian population-based study, Bernstein et al. compared 8,060 patients with IBD and 80,489 healthy controls matched for age, sex and area of residence. The incidence of arterial thromboembolic diseaseischemic heart disease, cerebrovascular disease and undifferentiated arterial thromboembolic disease-was compared in the two groups.

Males and females with any type of IBD, including Crohn's disease and ulcerative colitis, showed an increased risk of ischemic heart disease (incidence rate ratio 1.26, 95\% Cl 1.111.44). Only patients with Crohn's disease had an increased risk of cerebrovascular disease (incidence rate ratio $1.32,95 \% \mathrm{Cl} 1.05-1.66)$. The risk of undifferentiated arterial thromboembolic disease was also significantly increased in females of all ages with Crohn's disease, and in males with Crohn's disease aged 0-39 years and $40-59$ years.

This large, population-based study confirms that IBD of any type can increase the risk of cardiovascular disease in both sexes. Individuals with a diagnosis of Crohn's disease are at particular risk of cerebral arterial thromoboembolic problems. The authors speculated that smoking and genetic predisposition might contribute to their observed increase in risk.

Original article Bernstein CN et al. (2008) The incidence of arterial thromboembolic diseases in inflammatory bowel disease: a population-based study. Clin Gastroenterol Hepatol 6: 41-45 